\title{
TESTING FRAMEWORK FOR IMS-BASED IPTV
}

IPTV (Internet Protocol television) is one of the fast-growing communication services of the past years. Unfortunately, there is a lack of standardized deployed solutions and operators need to to rethink their position before upgrading IPTV solutions for next generation TV (HD TV and 3D TV). An efficient and up-to-date Interoperability Test Specification for IMS-Based IPTV is one of the opportunities for service providers and network operators to evaluate different features and services. TISPAN Release 3 covers many new use cases and features for IPTV (like user generated content, target advertising, etc.) and possible interactions with Voice/Data communications such as Social TV, Incoming Voice call management and notification on TV screen. In this paper, we will first provide a general overview of interoperability testing and its features. On an Instant Messaging use case, we will provide test descriptions including an end user test sequence as well as high level message flows at key standardized reference points in the TISPAN IMS-based IPTV infrastructure.

Key words: IPTV (Internet Protocol Television), interoperability, testing

\section{Introduction}

The current and future e-Communications market can be seen as a convergent multimedia market with an increasingly complex structure. Within this market we are faced with an unpredictable, sometimes fragmented, market development where potential barriers to achieve interoperability may be emerging. Additionally, within the present competitive environment, the risk of non-interoperability is increasing because of fast evolution of technology provided by different vendors, or the use of non-open standards.

Against this background there is an ever-increasing awareness of market players and regulators that mass-market development requires interoperability based on open standards. Additionally, the end-user appreciates more choice, but expects certainties.

Interoperability between devices, applications, data repositories, services and networks is a key requisite for taking advantage of the benefits of digital technologies. New standards are being created all the time, globally, and Europe's standard-setting framework must catch up with fast-moving technology markets if it is to remain relevant for the future. The main aim of standardization is to enable interoperability in a multi-vendor, multinetwork, multiservice environment. The absence of interoperability must not be the reason why final services for which there is great demand do not come into being.

With the introduction of IPTV, traditional wireline service providers and content providers are entering a new market delivering broadcast and $\mathrm{VoD}$ (Video on Demand) services to consumers. The success of this endeavor is heavily dependent upon how fast service providers can roll out reliable IPTV services that give con- sumers the most convenience and flexibility before competing providers do the same thing. Unfortunately, most of the deployed solutions are not standardized and operators will have to rethink soon their position before upgrading IPTV solutions for next generation TV (HD TV and 3D TV). An efficient and up-to-date Interoperability Test Specification for IMS-Based IPTV is one of the opportunities for service providers and network operators to evaluate different features and services. ETSI TISPAN Release 3 covers many new use cases and features for IPTV (like user generated content, target advertising, etc.) and possible interactions with Voice/Data communications such as Social TV, Incoming Voice call management and notification on TV screen. In this paper, we first provide a general overview of interoperability and its features. Then, we describe the ETSI TISPAN approach to IPTV. For one of them, the IMS-based IPTV, the testing framework is illustrated. Finally, on an Instant Messaging use case, we show test descriptions including an end user test sequence as well as high level message flows at key standardized reference points.

\section{Different Levels of Interoperability}

There is a number of definitions of the word interoperability: even at ETSI (European Telecommunications Standardization Institute) the term has different meanings in different contexts [1]. However, the following definitions are probably the closest to our understanding:

- "Interoperability is the ability of equipment from different manufacturers (or different systems) to communicate together on the same infrastructure (same system), or on another while roaming" from ETSI's Technical Committee TISPAN.

\footnotetext{
* Tatiana Kovacikova, Pavol Segec

Department of InfoCom Networks, Faculty of Management Sciences and Informatics, University of Zilina, Slovakia,

E-mail: tatiana.kovacikova@fri.uniza.sk
} 
Interoperability can be seen at different levels: technical interoperability, syntactical interoperability, semantic interoperability and organizational interoperability.

Technical interoperability is usually associated with hardware/ software components, systems and platforms that enable machine-tomachine communication to take place. This kind of interoperability is often centred on communication protocols and the infrastructure needed for those protocols to operate. Syntactical interoperability is usually associated with data formats, as messages transferred by communication protocols need to have a well-defined syntax and encoding, either in the form of bit-tables, or they can be represented using high-level transfer syntaxes such as HTML (Hypertext Markup Language), XML (eXtensible Markup Language) or ASN.1 (Abstract Syntax Notation). Semantic interoperability is usually associated with the meaning of content and concerns the human rather than machine interpretation of the content. Organizational interoperability is the ability of organizations to effectively communicate and transfer data and information even though they may be using a variety of different information systems over widely different infrastructures, possibly across different geographic regions and cultures. It can be said that organizational interoperability is the high-level issue and in general, it depends on successful technical, syntactical and semantic interoperability.

The development of standardized test specifications is an integral part of the ETSI strategy for ensuring interoperability. In the context of standardization ETSI focuses on the development of two types of test specifications, which reflect the principle: test the components first, then test the system, i.e.:

- Conformance test specifications; and

- Interoperability test specifications.

Conformance testing concentrates on specific components in a system, often related to a single standard (or a set of related standards). It is unit testing rather than system testing. Conformance testing is applied over open interfaces and checks for conformance to the requirements in a base specification or standard. Interoperability testing concentrates on a complete device or a collection of devices. It is system testing rather than unit testing. It is most commonly applied to end-to-end testing over networks. It shows, from the user's viewpoint, that functionality is accomplished, however, it does not show how.

Both, conformance as well as interoperability testing are important for successful deployment of the equipment from different vendors and its interworking [2]. The both should be regarded as complementary techniques. Many certification schemes require, for example, conformance testing as a prerequisite to interoperability testing (e.g. the Open Mobile Alliance or the WiMax Forum).

\section{TISPAN approach to IPTV}

While all major service providers are planning to launch or have already launched IPTV services, the strategies that will lead to the long-term economic success of these services are still being debated. A complex architecture is required to deliver competitive services, requiring close interoperability of all components involved in service delivery, including the Business Support System (BSS) and Operations Support System (OSS) and particularly the underlying network from the headend and VoD server to the home environment [3]. Within the home, there are other considerations in order to allow interworking of the STB with the control software (or middleware) and the integration of the middleware with other components (such as the headend, VoD servers, Web portals, and Electronic Program Guide (EPG)).

Aside from offering IPTV services, service providers have begun migrating their traditional fixed and mobile voice and communication services to converged IP NGN networks. As circuit-switched technology is phased out, new VoIP and rich media communication services are being deployed in packet-switched environments through the use of SIP signalling. IP Multimedia Subsystem (IMS) is emerging as an effective, standards-based architecture defining SIP-based voice and rich media service delivery. Today IMS is being promoted as the architecture of choice for multimedia communications services of all kinds. The ETSI and CableLabs ${ }^{\circledR}$ are creating standards to enable an IMS architecture to be supported on their specific access networks. ETSI Technical Committee TISPAN is the standards group within ETSI that is developing a more universal service delivery architecture that adapts the 3GPP-defined IMS standards to address the needs of wireline providers to build a policy- controlled IP transport network. Current versions of the IMS standards are focused on SIP-based communication services and PSTN replacement. Standards are still a work in progress, with $3 \mathrm{GPP}$ at Release 11 and TISPAN working on the definition of an NGN Release 3.

As part of its NGN (Next Generation Networks) Release 2 series of specifications, ETSI TISPAN has defined the integration of IPTV services in an NGN architecture to answer the needs of network service providers and equipment vendors. TISPAN defines two solutions for the integration of IPTV in the NGN architecture: Integrated IPTV subsystem and IMS-Based IPTV.

The Integrated IPTV subsystem [4] focuses on the integration of existing market solutions in an NGN environment. Network service providers benefit from the cost advantages of a Next Generation Network without strong modification of their current IPTV service. For instance, the NGN integrated IPTV subsystem reuses all applicable DVB-IPTV (Digital Video Broadcasting - IPTV technologies).

The IMS-based IPTV solution [5] allows blending of TV services with other telecommunication services (e.g. voice, presence, and data services). Network services providers can take full benefit of the IP Multimedia Subsystem (IMS) architecture while providing key end-user services. In this paper, we focus on this solution.

The deployment of IMS functionalities to support IPTV services enables using a number of interesting IPTV features [6], for example: 
- Integrated user registration and authentication (single sign-on);

- User subscription management;

- Session management, routing, service trigger, numbering;

- Interaction with existing NGN service enablers (presence, messaging, group management, etc.);

- Roaming and nomadism support;

- QoS and bearer control;

- Unified charging and billing.

The IMS based IPTV can also profit from additional features such as support for mobility, enabling interaction with existing NGN service enablers, service personalisation and media adaptation as well as to provide converged applications integrated voice, data video and mobile services to flexible quadruple play service concept.

The high-level functional architecture for the IMS-based IPTV is shown in Fig. 1.
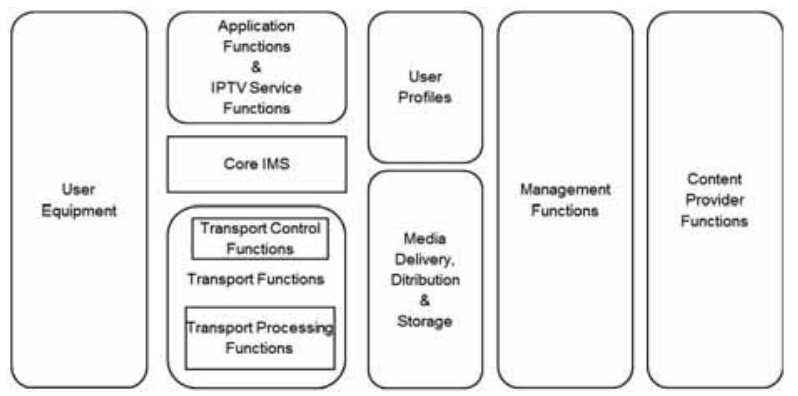

Fig. 1 High level functional architecture for the IMS-based IPTV

The IPTV enabled UE (User Equipment) terminates the IPTV control and media signals, and displays the corresponding information to the user. The UE interaction with the user allows selection of program, content, and service descriptions, such as content guides for broadcast and $\mathrm{VoD}$ services.

Application functions and IPTV service functions enable operation of or provide IPTV services. This includes IPTV Service Supporting Functions which represent common functions that could support or be used by other IPTV service or applications. Examples of IPTV service supporting functions may be Service Discovery and Selection functions.

User Profiles includes user data that are involved in providing IPTV services.

Core IMS provides functionality for authentication, authorization, and signalling for the setup of the service provisioning and content delivery. It routes signalling messages to the appropriate application server or triggers the applications based on settings maintained in the UPSF (User Profile Server Function). For resource reservation and admission control this function interacts with the RACS (Resource and Admission Control Subsystem).
Transport Functions include two kinds of functions:

- Transport Control: It contains functions from RACS and NASS (Network Attachment SubSystem). It provides policy control, resource reservation and admission control as well as IP address provisioning, network level user authentication and access network configuration as defined in TISPAN.

- Transport Processing Functions: They represent network access links and IP core. The IP core is in charge of data transmission with quality of service support.

The Media Delivery, Distribution and storage function receives and stores live feeds and media streams coming into the IPTV System from Content Providers. It is mainly in charge of media processing, delivery, storing, trans-coding and relaying. This function performs all these tasks along with the control of - or feedback to the IPTV Service and Control. Content protection may also be performed here or already protected content could be delivered over these functionalities.

The detailed specification of the architecture and functions of an IMS-based IPTV system is available in [5]. It should be noted that management functions and content provider functions are not specified in this document.

\section{IMS-Based IPTV - Testing Framework}

The IMS-based IPTV solution allows blending of TV services with other telecommunication services (e.g. voice, presence, and data services). Network services providers can take full benefit of the IMS architecture while providing key end-user services. Only a few basic use cases of IPTV have been defined in TISPAN Release 2. The IMS-based IPTV TISPAN Release 3 [7] covers many new use cases and features for IPTV (like user generated content, target advertising, etc.) and the possible interactions with Voice/Data communications such as Social TV, Incoming Voice call management and notification on TV screen. The IMS-Based IPTV Interoperability Test Specification for Release 2 is provided in the ETSI TS 186020 Ver. 2.1.1 [8] published in 2009. The new test cases have been recently developed and linked to TISPAN Release 3 within the ETSI STF 413, one of the authors of this paper was a member of [9]. The updated testing specification was published as the ETSI TS 186020 v.3.1.1 in July 2011 [10].

The specification covers the use of main IPTV functionality via different methods as defined in NGN Release 2 as well as NGN Release 3 new use cases and features for IPTV and possible interactions with Voice/Data communications such as Social TV, Incoming Voice call management and notification on TV screen. Interoperability test descriptions have been specified following the ETSI IPT test specification framework described in EG 202568 [11] and interoperability testing methodology defined in EG 202237 [12], i.e. interoperability testing with a conformance relation. Each interoperability test description includes an end user test sequence as well as a table for checking of high level message flows at key 
standardized reference points in the TISPAN IMS-based IPTV infrastructure [5] and [7].

Fig. 2 shows the abstract test architecture of an IMS-based IPTV system based on the general IPTV architecture. In this figure, each node groups different IPTV logical functions. Interfaces within each node are considered internal and not taken into account in conformance criteria. Reference points (Ut, e2 and y2 towards BC-MCF) in dotted line are not in the scope of the specification. It should be noted that in a real IMS-based IPTV system some of the nodes shown in Fig. 2 may also be collocated in the same equipment. In this case it is however still assumed that their connecting interfaces are still available for monitoring purposes.

Each node framed with a solid line is considered Equipment under Test (EUT) in the context of the ETSI interoperability testing methodology [12]. The collection of all EUTs makes up the System Under Test (SUT). Dashed nodes indicate other equipment, i.e. support nodes, required to execute at least some of the tests. The latter nodes are considered not to be part of the SUT.

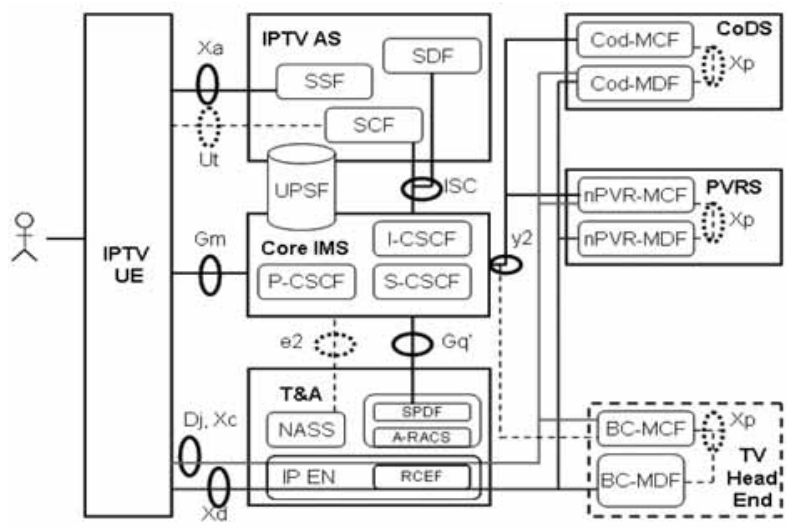

Fig. 2 IMS-based IPTV test architecture

\begin{tabular}{|c|c|c|}
\hline \multicolumn{3}{|r|}{ Interoperability Test Description } \\
\hline Identifier: & \multicolumn{2}{|c|}{ TD_IMS_IPTV_IM_0001 } \\
\hline Summary: & \multicolumn{2}{|r|}{ User sends an instant message through OMA Instant Messaging } \\
\hline References: & \multicolumn{2}{|r|}{ ETSI TS 182027 [1] clause 9.3.1; ETSI TS 183063 [2] clauses 5.1.17.1 \& 5.3.16.1 } \\
\hline Configuration: & \multicolumn{2}{|r|}{ CF_IMS_IPTV } \\
\hline Required Equipment: & \multicolumn{2}{|c|}{ IPTV aware UE, Core IMS, IPTV AS } \\
\hline Pre-test conditions: & \multicolumn{2}{|c|}{$\begin{array}{l}\text { - UE supports OMA Instant Messaging } \\
\text { - UE is registered in Core IMS using userIPTV_priv identity }\end{array}$} \\
\hline \multirow[t]{4}{*}{ Test Sequence: } & Step & \\
\hline & 1 & User registers to OMA Instant Messaging service \\
\hline & 2 & User sends “Available soon?” IM \\
\hline & 3 & Verify that UE receives SIP $200 \mathrm{OK}$ \\
\hline \multirow[t]{2}{*}{ Conformance Criteria: } & Check & \\
\hline & 1 & Message exchange follows the below table \\
\hline
\end{tabular}

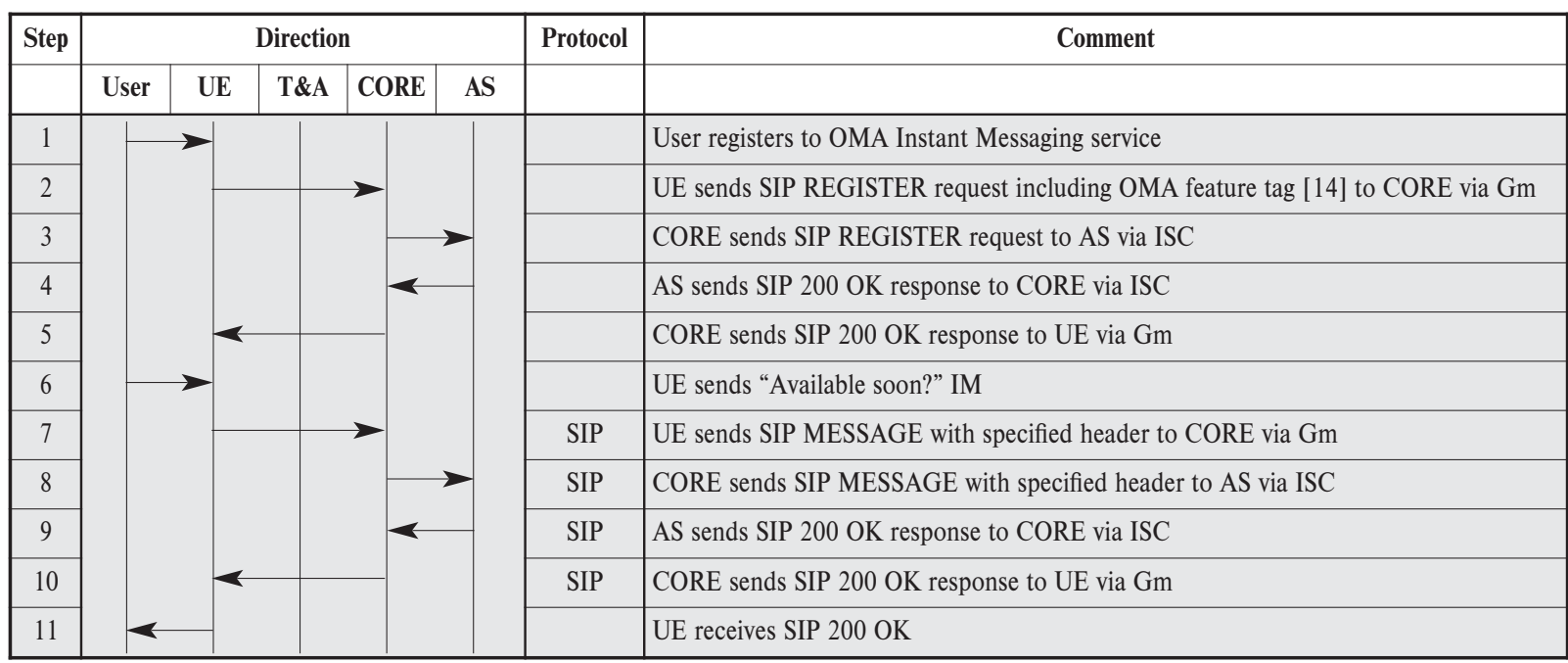

Fig. 3 Interoperability test description for Instant messaging sending 
In the ETSI TS 186 020, the IMS-based IPTV interoperability test descriptions (TD) for systems composed of equipment by different vendors are defined. Each TD includes a test sequence describing user interactions with IPTV equipment as well as messages exchanged between IPTV equipment at selected standardized reference points.

\section{Test Description - Instant Messaging Example}

Instant messaging, often shortened to simply "IM" or "IMing," is the exchange of text messages through a software application in real-time. In this clause, we provide test description for Instant messaging in the context of TISPAN IPTV. On this example, we show how the IMS-based IPTV procedures with other IMS services are to be tested. The IPTV UE shall support OMA Instant Messaging according to [13]. It should be noted that SIP messages as 100 TRYING are not included in sequence diagrams below.

Interoperability test description for Instant messaging sending is illustrated in Fig. 3.

Interoperability test description for Instant messaging receiving is illustrated in Fig. 4.

\section{Conclusion}

IMS is expected to respond to and solve many of the industry's biggest technological challenges, including the lack of interoperability among operators who offer the same services and the inability of operators to take advantage of converged networks. IMS is a key component of the Next-Generation Networks and IPTV has been identified as one of the first services to be merged with Voice/Data communications on IMS architecture.

Only a few basic use cases of IPTV have been defined in TISPAN Release 2. On the other hand, TISPAN Release 3 covers many new use cases and features for IPTV and the possible interactions with Voice/Data communications such as Social TV, Incoming Voice call management and notification on TV screen. As a consequence, the update of the IMS-Based IPTV Interoperability Test specifications is an important step towards the fully inter= operable IPTV solution.

In this paper, we described the ETSI TISPAN approaches to IPTV. For one of them, the IMS-based IPTV, the testing framework was described. We selected the IM use case for test descriptions including an end user test sequence as well as high level message flows at key standardized reference points. Further work is still required to cover and provide test descriptions for all Release 3 features.

\begin{tabular}{|c|c|c|}
\hline \multicolumn{3}{|r|}{ Interoperability Test Description } \\
\hline Identifier: & \multicolumn{2}{|c|}{ TD_IMS_IPTV_IM_0002 } \\
\hline Summary: & \multicolumn{2}{|c|}{ User receives an instant message through OMA Instant Messaging } \\
\hline References: & \multicolumn{2}{|r|}{ ETSI TS 182027 [1] clause 9.3.1; ETSI TS 183063 [2] clauses 5.1.17.1 \& 5.3.16.1 } \\
\hline Configuration: & \multicolumn{2}{|r|}{ CF_IMS_IPTV } \\
\hline Required Equipment: & \multicolumn{2}{|c|}{ IPTV aware UE, Core IMS, IPTV AS } \\
\hline Pre-test conditions: & \multicolumn{2}{|c|}{$\begin{array}{l}\text { - UE supports OMA Instant Messaging } \\
\text {-UE is registered in Core IMS using userIPTV_priv identity } \\
\text { - UE is registered to OMA IM service (see TD_IMS_IPTV_IM_0001) }\end{array}$} \\
\hline \multirow[t]{3}{*}{ Test Sequence: } & Step & \\
\hline & 1 & An Instant Message is required to be sent to UE \\
\hline & 2 & Verify that UE displays the received IM \\
\hline \multirow[t]{2}{*}{ Conformance Criteria: } & Check & \\
\hline & 1 & Message exchange follows the below table \\
\hline
\end{tabular}

\begin{tabular}{|c|c|c|c|c|c|c|c|}
\hline Step & \multicolumn{5}{|c|}{ Direction } & Protocol & Comment \\
\hline & User & UE & T\&A & CORE & AS & & \\
\hline 1 & & & & & $<$ & & An Instant Message is required to be sent to UE \\
\hline 2 & & & & $<$ & & & AS sends SIP MESSAGE to CORE via ISC \\
\hline 3 & & & & & & & CORE sends SIP MESSAGE to UE via $\mathrm{Gm}$ \\
\hline 4 & & & & & & & UE sends $200 \mathrm{OK}$ to CORE via $\mathrm{Gm}$ \\
\hline 5 & & & & & & & CORE sends $200 \mathrm{OK}$ to AS via ISC \\
\hline 6 & $<$ & & & & & & Verify that UE displays the received IM \\
\hline
\end{tabular}

Fig. 4 Interoperability test description for Instant messaging receiving 


\section{References}

[1] VAN DER VEER, H., WILES, A.: ETSI White Paper No. 3, Achieving Technical, Interoperability - the ETSI Approach, $3^{\text {rd }}$ edition April 2008, http://www.etsi.org/WebSite/document/whitepapers/IOP\%20whitepaper\%20Edition\%203\%20final.pdf

[2] MONKEWICH, O.: Conformance and Interoperability Testing Tutorial, ITU-T SG 17, http://www.itu.int/dms_pub/itu-t/oth/15/04/ T15040000080001PDFE.pdf

[3] Cisco White Paper: The Evolving IPTV Service Architecture, http://www.cisco.com/en/US/solutions/collateral/ns341/ns525/ns537/ ns549/ns746/net_implementation_white_paper0900aecd806530a4.pdf

[4] ETSI TS 182 028: Telecommunications and Internet converged Services and Protocols for Advanced Networking (TISPAN); NGN integrated IPTV subsystem Architecture, 2011

[5] ETSI TS 182 027: Telecommunications and Internet converged Services and Protocols for Advanced Networking (TISPAN); IPTV Architecture; IPTV functions supported by the IMS subsystem, 2011

[6] MIKOCZY, E., SIVCHENKO, D., RAKOCEVIC, V.: IMS based IPTV services - Architecture and Implementation, Proc. of the $3^{\text {rd }}$ intern. conference on Mobile multimedia communications, ICST, Brussels, Belgium, 2007

[7] ETSI TS 183063 (V3.5.2): Telecommunications and Internet converged Services and Protocols for Advanced Networking (TISPAN); IMS-based IPTV stage 3 specification

[8] ETSI TS 186020 v.2.1.1: Telecommunications and Internet converged Services and Protocols for Advanced Networking (TISPAN); IMS-based IPTV interoperability test specification, 2009

[9] http://portal.etsi.org/STFs/STF_HomePages/STF413/STF413.asp

[10] ETSI TS 186020 v.3.1.1: Telecommunications and Internet converged Services and Protocols for Advanced Networking (TISPAN); IMS-based IPTV interoperability test specification, 2011

[11] ETSI EG 202 568: Methods for Testing and Specification (MTS); Internet Protocol Testing (IPT); Testing: Methodology and Framework

[12] ETSI EG 202 237: Methods for Testing and Specification (MTS); Internet Protocol Testing (IPT); Generic approach to interoperability testing

[13] OMA-TS-SIMPLE-IM-V1-0-20100322-C: OMA: Instant Messaging using SIMPLE. 